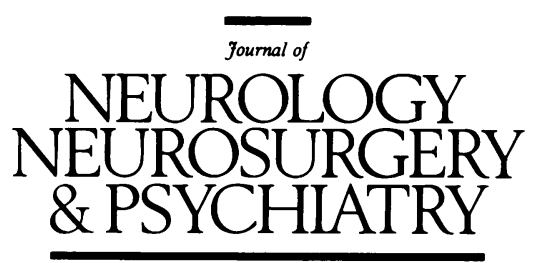

\title{
Behaviour changes following temporal lobectomy, with special reference to psychosis
}

The severe behaviour consequences of bilateral extirpation of the temporal lobes were realised in the earliest reports of temporal lobe resection, and several case studies or series were published in the early 1950 s. $^{1}$ The operations, which included amygdalotomy, uncotomy, and medial temporal lobotomy were initially carried out for the relief of psychosis, mainly in schizophrenic patients, but some patients with epilepsy were included. The severe memory deficits which resulted were not seen with unilateral operations, which became the accepted approach.

In contrast to the recognised need for proper psychometric evaluation before surgery for epilepsy, the necessity for adequate psychiatric assessment was not (and has not been) realised in many centres. The importance of such evaluations emerged early in the history of these operations. Thus the number of patients with preoperative psychiatric abnormalities was far greater in the Falconer Guy's-Maudsley series than in that from Penfield at Montreal. Although this largely reflected referral patterns, at some stage the Montreal group specifically excluded from the operation patients with gross mental changes. $^{2}$

In the early studies, it was agreed that some patterns of behaviour could change after temporal lobectomy, especially aggressive problems, while others were not influenced or deteriorated. Improvements were not immediate, and could occur slowly over a period of 12 months or longer. ${ }^{3}$ Green et $a l^{4}$ were more explicit, noting that patients with constant hostility benefitted from the operation, while those with psychotic behaviour did not.

The early experiences of Falconer et al were written up in $1957 .^{2}$ The post-operative follow up was two to five years, and included information from rating scales, home visits and relatives. Post-operative scores were improved in eighteen cases, unchanged in three and declined in five. All five of the latter continued to have seizures. There was a reduction in outwardly expressed aggressiveness and an increase of depressive mood swings (eleven cases), the latter sometimes so severe that readmission to hospital was necessary; ECT was given to five patients. These depressive states tended to recur for up to 18 months and were not related to post-operative seizures.

Another early series was that of Simmel and Counts ${ }^{5}$ from Illinois. Forty patients were followed up after five years, and eleven were seizure free. Most patients had some personality disturbance or psychoses before surgery, although this was not well documented. They state "patients reported to talk about receiving messages from God, about their food being poisoned, etc, were classified as psychotic". Post-operative assessment was based on interviews with patients and their families, and patients were rated as "good", "marginal", or "psychotic".

Pre-surgery, 21 were "marginal", and 11 "psychotic". At the time of follow up, 19 were "marginal" and 14 "psychotic". In terms of change, two were improved, 22 were about the same while 16 deteriorated. Of the 14 "psychotic" patients, 10 were definitely psychotic before the operation, while six had "gone further downhill since surgery." Four new cases emerged post-operatively.

Falconer, in discussing the paper of Simmel and Counts said: ". . the idea might get abroad that you cannot relieve such symptoms by temporal lobectomy ... . seven (of my psychiatric patients) had frank psychotic episodes, and three of them are now better . . . I feel at times psychiatric disturbances can be relieved in association with temporal lobectomies."

\section{The Maudsley series}

Falconer, Taylor and their colleagues ${ }^{7-11}$ reviewed in a series of papers the consequences of temporal lobectomy on behaviour in an extended series of over 200 patients having unilateral temporal lobectomy; follow up was from six to twenty years. Nearly $50 \%$ of the cases had mesial temporal sclerosis, hamartomas accounted for one fifth to one quarter, one tenth had miscellaneous lesions such as scars or infarcts, while no specific lesion was found in the rest. In the first series of 100 cases,${ }^{8}$ there were twelve with confusional states, two with sudden religious conversions, six were diagnosed as paranoid, while three were schizophrenic. There were two suicides post-operatively. Those with paranoid or affective conditions pre-operatively had less prominent delusions post-operatively, while the schizophrenic group did poorly. Patients in a third group, with intermittent symptoms, often ictally linked, did rather better.

In the second better documented series of 100 cases $^{9}$ sixteen were psychotic pre-operatively. Eight had schizophreniform psychoses, five paranoid hallucinatory psychoses, one simple schizophrenia and one catatonia. One was referred to as "organic psychosis". Post-operatively there were nineteen psychotics. Four patients had lost their psychoses, while seven developed psychoses. Of the whole sample, only thirteen had been considered psychiatrically normal before operation. On a social adjustment scale, $61 \%$ were reported as improved, $28 \%$ worse and $11 \%$ the same when pre- and post-operative scores were compared. The main improvements concerned family and extra familial relationships and work status. Those patients with "good" post-operative adjustment showed an excess of normal or 
neurotic personalities before surgery, while those who fared worst had an excess of psychopathic disorder or psychoses. The latter also had a lower incidence of mesial temporal sclerosis, and an increase in non-specific lesions on pathological examination of the resected specimen. Complete relief of seizures was not essential for good adjustment, and only $60 \%$ of those who moved from "bad" to "good" social adjustment with the operation became seizure free. They also reported on aggressive behaviour. Pre-operatively, aggressive behaviour was as common with mesial temporal sclerosis as with hamartomas, but postoperatively, patients with mesial temporal sclerosis improved the most. In this series there were five suicides.

Seven of Falconer's psychotic cases were in a series of eleven cases of schizophrenia-like psychoses that had been operated on and reported by Glithero and Slater. ${ }^{12}$ Schizophrenic symptoms receded in only three, but in another five they were reported as improved post-operatively.

In an extended follow up study, (five to 24 years) Taylor and March $^{13}$ examined post-operative deaths in 193 temporal lobectomy patients who had a potential survival of at least five years. Four patients died from tumours, seven from natural causes, eight in status, and three in accidents. There were nine suicides $(4.6 \%)$ and six deaths in "unclear" circumstances. The interval between operation and death was shorter in those who committed suicide or whose mode of death was unclear, compared with the rest. Interestingly, the mortality in the first two years postoperatively was double that in any subsequent two year period.

The Maudsley series has recently been the subject of another follow up investigation reported by Bruon et al. ${ }^{14} 15$ They examined the notes and pathological specimens from 249 cases operated on from 1950. They assessed fit frequency and social adjustment, both graded into four categories ranging from greatly improved to worse. The former included only those fit-free for five years, and the social adjustment was based on items, such as, return to work, subsequent marriage, living away from institutional care; special attention was given to depression, aggresion, and schizophrenia.

Of 27 deaths, they reported six suicides $(2 \cdot 4 \%)$ which occurred at an average time of 5.6 years after the operation. The results of surgery on personality and social adjustment in 234 cases revealed $59(25 \%)$ to be greatly improved, 53 $(22.6 \%)$ improved, $73(31.2 \%)$ unaltered and $49(20.9 \%)$ were worse. Again it was found that the Ammon's horn sclerosis group had the best chance of improvement, while those with no abnormality were made significantly worse by the operation.

There was a substantial increase in depression postoperatively. While only one case was rated depressed preoperatively, $24(10 \%)$ had post operative affective disorder. This was more common in those with Ammon's horn sclerosis, double pathology and no apparent pathology. All six suicides were in this series of 24 , but not associated with any special pathology. Aggression improved, being assessed as present in $22 \%$ pre-operatively and $16 \%$ post-operatively. This was best in both those with "alien tissue" (lesions with abnormal or "alien" tissue elements associated with them), and the Ammon's horn sclerosis groups.

In the total series, 25 patients had either a pre- or a postoperative case note diagnosis of schizophrenia. Of sixteen with a pre-operative diagnosis of schizophrenia eleven had a left and five a right sided focus. Post-operatively the figures were five and four respectively. The number of cases with gangliogliomas (4) who developed psychosis was unexpectedly large $(44 \%)$. Further, there were four patients who lost their psychosis after the operation, three with Ammon's horn sclerosis, and one with alien tissue, all being left sided lobectomies.

There are several important conclusions that emerge from these data. First it seems that schizophrenia-like states do not emerge at random in temporal lobe epilepsy. They are associated with medial temporal pathology, often but not exclusively with alien tissue lesions as Taylor ${ }^{11}$ suggested. Secondly, there is an over-representation of left sided pathology, as noted by Taylor, but the relationship holds only for assessment pre-operatively. In postoperative status there is bias towards right sided lesions increasing the vulnerability to develop a psychosis, left sided lesions favouring amelioration. Thirdly, gangliogliomas may have a special relationship to psychosis.

Finally, the data Burton analysed reveal the dissociation between the effect of temporal lobectomy on seizures, and on behavioural adaptation. It is often assumed that a decrease of seizures is automatically associated with improved behaviour, but this is not the case. Patients with Ammon's horn sclerosis did well with both frequency of fits and social adjustment. In contrast, however, the removal of mixed glial and neuronoglial lesions helped seizures but not social adjustment. Patients with trauma or indefinite pathology tended to have seizures unaffected, and their social adjustment often became worse.

\section{Other series}

The state of the art in 1975 was noted in a review by Jensen $^{16}$ of follow up psychiatric status from five centres, including the Maudsley. She noted a marked divergence of results, especially regarding the unchanged/deteriorated category. This may reflect on selection, or on the adequacy of the evaluations. On summing the different series, Jensen noted that $23.5 \%$ were considered mentally normal at the time of follow up, compared with $6.2 \%$ before operation. In contrast, $35.6 \%$ were unchanged or deteriorated and $40.9 \%$ were improved.

Further data on psychosis from the Danish series were given by Jensen et al. ${ }^{1718}$ They surveyed 74 patients who had received unilateral temporal lobectomy, $45(61 \%)$ of whom were seizure free post-operatively. There were twenty psychotic patients, either pre- or post-operatively. Seventeen had partial seizures with secondary generalisation. Eleven were psychotic pre-operatively. Eight improved and one recovered after surgery. Nine patients became psychotic post-operatively, and in six there was complete relief of seizures. Curiously the important issue of laterality is glossed over by the statement that "operations on the right or on the non-dominant sides were more frequent in the psychotic patients ...', but no figures were given.

Relief of seizures was the most important factor in determining rehabilitation to work, but there was no obvious relationship between post-operative seizure frequency and psychiatric status. Behavioural disturbances were the most improved by operation, and this finding agrees with other series. All suicide attempts occurred within the first post-operative month.

This series is of importance in confirming that psychosis can arise de novo post-operatively, and that this is seen in spite of or because of seizure relief. One speculation which emerges is that at least in some of these cases a mechanism similar to that of forced normalisation is operative.

Other groups have provided limited information on the behavioural changes of patients having temporal lobectomy. In a study of patients with psychosis that had undergone lobectomy, Sherwin ${ }^{19}$ noted seven cases rendered seizure free in whom no change in psychosis was observed. Walker and Blumer ${ }^{20}$ provided data on 50 patients. Irritability, anger and rage, noted in 29 patients 
pre-operatively, improved in 22, although six patients developed such behaviours. There were nine patients with psychosis; one was alcohol related. Six cases developed a schizophrenia-like psychosis post-operatively, in three this occurred some time after the operation. No data on pathology or laterality were given, and the general conclusion drawn was that temporal lobectomy had little or no effect on psychosis associated with epilepsy.

Steven ${ }^{21}$ followed a personal series of fourteen cases for 25-30 years, six of whom were psychiatrically well, nine being seizure free. However, six became psychiatrically worse post-operatively, three of whom were reported as normal before surgery. No less than five developed a paranoid psychosis, four of whom had a right sided operation. The majority were not seizure free.

Polkey ${ }^{22}$ has given data on a new Maudsley series of forty patients, seventeen of whom had some form of mental disorder pre-operatively. Aggression was the commonest, and there were no psychotic patients. Post-operatively two developed psychosis, and Polkey describes a third not in the series of forty. All had non-dominant resections, and in one who subsequently died in an accident, the opposite temporal lobe was pathologically normal. In another, the psychosis resolved when the patient's seizures returned.

\section{Conclusions}

These data on the psychiatric consequences of temporal lobectomy lead to several conclusions. First, most centres, including the Maudsley hospital group, have now stopped operating on floridly psychotic patients. This seems largely based on the observation that psychosis generally does not improve with the operation. ${ }^{81718}$ Whether or not this course of action is justified is not clear. It might still be considered better to be psychotic without seizures than to be psychotic with them. This policy, however, has not been taken to its logical conclusion, namely that of a comprehensive psychiatric evaluation being carried out as a routine pre-operatively. This is in marked contrast to the insistence, correctly, on good psychological assessment.

It appears that the psychosocial adjustment of patients post-operatively is by no means as good as the results on seizures, and in some series as many patients deteriorate as improve. The improvements in behaviour generally recorded are those of diminished aggression, irritability and disturbed conduct, and these seem more likely to improve with improvement of seizures and a pathological diagnosis of mesial temporal sclerosis. In contrast, post-operative depression, and both early and late suicide are reported from several groups, which may be seen as a complication of the surgery. Some authors suggest this may be no more than a reflection of the high frequency of depression in nonoperated epileptic patients. The figure of $10 \%$ of patients developing depression, ${ }^{13}$ and suicide rates of up to $5 \%$ emphasise the need for continuing psychiatric observation of patients that have had operations. This is not done in most centres. Indeed, the number of good follow up series of psychiatric data is abysmal, considering the number of centres now doing such procedures.

Psychoses, paranoid or schizophrenia-like in nature, develop de novo post-operatively in 3.8 to $35.7 \%$ (mean $7 \cdot 6 \%)^{5}{ }^{514171820-22}$ This emphasises the importance of postoperative behavioural assessments, and continuing supervision of patients. There are no clear predictors of the patients that will develop the psychoses, and neither is there agreement on the relationship to the seizure control. There is nonetheless the suggestion that the phenomenon of forced normalisation may be operative in at least some cases. It could be argued that the patients would have developed psychosis in any case, supported by a high genetic loading for psychiatric illness in the series by Jensen and Larsen. ${ }^{19}$ This aspect not been commented on by other authors.

An interesting finding, which emerges from the new cases of psychosis post-operatively, is that where laterality has been established, it is right sided in over $60 \%$ of cases in the later series. Bruton even suggested a link to a specific pathology, namely gangliogliomas. That there may be an association with right sided operations is supported further by reports of Mace and Trimble, ${ }^{23}$ in which six consecutive patients are described who developed a psychosis following temporal lobectomy; all had right sided operations.

In summary, it is suggested that psychoses do emerge de novo post operatively, and there is some evidence of an increase in depression. However, the phenomenology of these states has yet to be well established. Suicide may be one complication of the procedure. Since psychosis does not develop at random in patients suffering from temporal lobe epilepsy and probably does not do so in new postoperative cases, closer attention to the psychiatric status of patients before and after temporal lobectomy would be rewarding.

Raymond Way Neuropsychiatry Research Group,

CHAEL R TRIMBLE Institute of Neurology,

National Hospital for Neurology and Neurosurgery, London, UK or total removal of the temporal lobes in man. In: Baldwin M, Bailey P, eds. or total removal of the temporal lobes in man. In: Baldwin M, Bailey
Temporal lobe epilepsy. Springfield, IIl: Thomas CC, 1958:510-29.

2 Hill D, Pond DW, Mitchell W, Falconer MA. Personality changes following temporal lobectomy for epilepsy. J Mental Sci 1957;103:18-27.

3 Penfield W. Discussion. In: Baldwin M, Bailey P, eds. Temporal lobe epilepsy. Springfield, Ill: Thomas CC, 1958:484-5.

4 Green JR, Duisberg REH, McGrath WB. Focal epilepsy of psychomotor type: a preliminary report on the observations on effects of surgical therapy. J Neurosurg 1951;8:157-79.

5 Simmel ML, Counts S. Clinical and psychological results of anterior temporal lobectomy in patients with psychomotor epilepsy. In: Baldwin M, Bailey P, eds. Temporal lobe epilepsy. Springfield, Ill: Thomas CC,

6 Falconer M. Discussion. In: Baldwin M, Bailey P, eds. Temporal lobe epilepsy. Springfield, Ill: Thomas CC, 1958:537-58.

7 Falconer MA. Surgical treatment of drug resistant epilepsy due to mesial temporal sclerosis. Arch Neurol 1973;19:353.

8 Serafetinides EA, Falconer MA. The effects of temporal lobectomy in epileptic patients with psychosis. J Ment Sci 1962;108:585-93.

Taylor DC. Mental state and temporal lobe epilepsy. Epilepsia 1973;13: 727-65.

10 Taylor DC, Falconer MA. Clinical, socio-economic and psychological changes after temporal lobectomy for epilepsy. Br J Psychiat 1968;114:1247-61.

11 Taylor DC. Factors influencing the occurrence of schizophrenia-like psychosis in patients with temporal lobe epilepsy. Psychol Med 1975;5: 249-54.

12 Glithero E, Slater E. Follow up record and outcome. Br J Psychiat 1963;109:134-42.

13 Taylor DC, Marsh SM. Implications of long term follow up studies in epilepsy. In: JK Penry, ed. Epilepsy: 8th International Symposium. Raven

14 Bruton CJ. The neuropathology of temporal lobe epilepsy (Maudsley Monograph 31). Oxford: Oxford University Press, 1988.

15 Roberts GW, Done DJ, Burton C, Crow TJ. A "mock-up" of schizophrenia: temporal lobe epilepsy and schizophrenia-like psychosis. Biol Psychiat 1990;28:127-43.

16 Jensen I. Temporal lobe surgery around the world. Acta Neurol Scand 1975;52:354-73.

17 Jensen I, Larsen JK. Mental aspects of temporal lobe epilepsy. J Neurol Neurosurg Psychiatry 1979;42:256-65.

18 Jensen I, Larsen JK. Psychoses in drug resistant temporal lobe epilepsy. J Neurol Neurosurg Psychiatry 1979;42:948-54.

19 Sherwin I. Psychosis associated with epilepsy significance of laterality of the epileptogenic lesion. J Neurol Neurosurg Psychiatry 1981;44:83-5.

20 Walker EA, Blumer D. Behaviour effects of temporal lobectomy. In: Blume D, ed. Psychiatric aspects of epilepsy. Washington: American Psychiatric

21 Stevens JR. 1990 Psychiatric consequences of temporal lobectomy for intractible seizures: a 20-30 year follow up of fourteen cases. Psychol Med intractible seizur.

22 Polkey CE. Effects of anterior temporal lobectomy apart from the relief of seizures. J Roy Soc Med 1983;76:354-8.

23 Mace C, Trimble MR. Psychoses following temporal lobe surgery: a report of six cases. J Neurol Neurosurg Psychiatry 1991;54:639-44. 